\title{
RELEVANCIA DEL NÚMERO Y COMPOSICIÓN DE SECUENCIAS DE LOS NUDOS CROMOSÓMICOS EN LA CARACTERIZACIÓN DE MAÍZ Y TEOCINTLE
}

\section{RELEVANCE OF NUMBER AND SEQUENCE COMPOSITION OF KNOBS IN THE CHARACTERIZATION OF MAIZE AND TEOSINTE}

\author{
Graciela Esther González ${ }^{\star}$ María Florencia Fourastié y Lidia Poggio
}

\begin{abstract}
Departamento de Ecología Genética y Evolución, Facultad de Ciencias Exactas y Naturales, Universidad de Buenos Aires. Intendente Güiraldes 2160 - Ciudad Universitaria - C1428EGA. Ciudad Autónoma de Buenos Aires, Argentina. Tel: 011-4576-3300 int. 218.

${ }^{*}$ Autor para correspondencia (gegonzalez@ege.fcen.uba.ar)
\end{abstract}

\section{RESUMEN}

Los knobs o nudos cromosómicos son bloques heterocromáticos que se pueden encontrar en 34 posiciones cromosómicas diferentes en maíz (Zea mays ssp. mays) y teocintle (Zea mays ssp. parviglumis, Zea mays ssp. mexicana, Zea mays ssp. huehuetenanguensis, Zea luxurians, Zea nicaragüensis y Zea diploperennis). Estos son variables en número, tamaño y posición entre accesiones de maíz y pueden detectarse, mediante bandeos cromosómicos C y DAPI (4', 6'-diamino2 -fenilindol), tanto en metafases como en núcleos interfásicos. A nivel molecular están constituidos por dos familias de secuencias (180-pb y TR-1) representadas por miles a millones de copias, en distintas proporciones unas de otras, y constituyen diferentes tipos de knobs. En este trabajo se analizaron las variaciones en la cantidad de regiones knob y en la composición de sus secuencias en distintas accesiones de maíz y teocintle, para su caracterización citogenética. Para ello se emplearon técnicas de citogenética clásica (tinción con DAPI) y molecular (Hibridación In Situ Fluorescente-FISH). El fluorocromo DAPI reveló variación en el número de regiones DAPI+ (knobs) sobre los cromosomas y los núcleos interfásicos. El FISH mostró que las señales de hibridación se localizaron en las regiones DAPI+ y reveló la constitución de secuencias de cada knob, para así demostrar que no sólo existe variación en el número de knobs entre las accesiones analizadas sino también en el tipo de secuencias que los componen. Con los patrones de hibridación obtenidos fue posible diferenciar entre las accesiones de maíz y de teocintle estudiadas, aportar datos para su caracterización citogenética, y contribuir al conocimiento de la variabilidad genética del género Zea. FISH

Palabras clave: Zea, knobs, maíz, teocintle, caracterización citogenética,

\section{SUMMARY}

Knobs are heterochromatic blocks that can be found in $\mathbf{3 4}$ different chromosomal locations in maize (Zea mays ssp. mays) and teosintes (Zea mays ssp. parviglumis, Zea mays ssp. mexicana, Zea mays ssp. huehuetenanguensis, Zea luxurians, Zea nicaragüensis and Zea diploperennis). These are variable in number, size and position among different accessions of maize and teosintes and can be detected by chromosome banding C and DAPI (4', 6'-diamidino-2-phenylindole), both in methaphases and in interphase nuclei. At a molecular level they are constituted by two families of sequences (180-bp and TR-1), and are represented by thousands to millions of copies, in different proportions to each other, forming the different types of knobs. In this study we analyzed if the variation in the number of knobs regions and the composition of their sequences allow the cytogenetic characterization of different maize and teosinte accessions. For this study, we applied classical (DAPI) and molecular (Fluorescence In Situ HybridizationFISH) cytogenetic techniques. DAPI staining showed variations in the number of DAPI+ regions (knobs) on chromosomes and interphase nuclei. FISH showed co-localized hybridization signals with the DAPI+ regions; it also revealed variation of the sequences composition of knobs among the accessions tested. These results allowed finding differential hybridization patterns to discern different accessions of maize and teosintes herein studied, therefore providing data for cytogenetic characterization and contributing to the knowledge of the genetic variability of the genus Zea.

Index words: Zea, knobs, maize, teosintes, cytogenetic characterization, FISH.

\section{INTRODUCCIÓN}

El género Zea L. (Poaceae/Tribu Maydeae) es una de las entidades biológicas más importantes, ya que el maíz (Zea mays ssp. mays) es cultivado en toda la Tierra y es en la actualidad una de las principales fuentes de alimentación humana y animal (Cámara y Arancibia, 2007). A las especies silvestres de Zea se les denomina colectivamente teocintle. Este grupo comprende tres subespecies de Zea mays L. (Zea mays ssp. parviglumis Iltis \& Doebley, Zea mays ssp. mexicana Schrader, y Zea mays ssp. huehuetenanguensis Iltis \& Doebley), y cuatro especies: Zea luxurians (Durieu \& Ascherson) Bird, Zea nicaragüensis (Iltis \& Benz), Zea diploperennis Iltis (Doebley \& Guzmán) y Zea perennis (Reeves \& Mangelsdorf). Todos los taxones mencionados poseen $2 \mathrm{n}=$ 20 cromosomas excepto Zea perennis que posee $2 n=40$. En algunas razas de maíz se han observado cromosomas accesorios (cromosomas B) en frecuencias variables (Rosato et al., 1998; Poggio et al., 1998).

Los cromosomas de Zea presentan bloques de heterocromatina, denominados nudos cromosómicos o knobs (Kato, 1976; McClintock, 1978). Esta heterocromatina posee mayor condensación y está constituida por secuencias de ADN altamente repetidas (Peacock et al., 1981). Los knobs pueden visualizarse tanto en células en división como también en núcleos interfásicos como cromocentros (Wan y Widholm, 1992). En núcleos interfásicos los bloques heterocromáticos se corresponden con los knobs, y existe una correlación positiva entre el número y tamaño de estos bloques y las bandas heterocromáticas de los cromosomas metafásicos (Kato, 1976). Este último autor observó, en paquitenos de maíz y teocintle, que los knobs pueden encontrarse en 34 posiciones cromosómicas diferentes, y se pueden detectar 
mediante bandeos cromosómicos C y DAPI (Poggio et al., 1998; González y Poggio, 2011).

Algunos estudios demostraron que los knobs son variables en tamaño, número y posición entre diferentes líneas y razas de maíz (Kato, 1976; McClintock et al., 1981; Tito et al., 1991; Rosato et al., 1998). Estos caracteres cariotípicos han sido utilizados como marcadores en la caracterización de distintas líneas y razas de maíz mexicanas y centroamericanas (Kato, 1976; McClintock et al., 1981). Lo anterior demuestra que el estudio de los nudos cromosómicos puede ser una herramienta útil para la clasificación racial y geográfica de las razas de maíz y una indicación de diversidad racial (McClintock et al., 1981).

Existen diferencias intra e interespecíficas en el tamaño del genoma de maíz y de teocintle que fueron relacionadas con determinados factores ecogeográficos, atribuidas principalmente a las variaciones en el número y tamaño de los knobs (Laurie y Bennett, 1985; Rayburn et al., 1985; Tito et al., 1991; Poggio y Naranjo, 1990). Poggio et al. (1998) observaron que el número, tamaño y frecuencia de knobs poseen una correlación negativa con la altitud de cultivo de distintas razas de maíz nativas del noroeste de Argentina (NOA). Aún se desconocen las razones, causas o mecanismos evolutivos que expliquen estas correlaciones, y si las mismas pueden depender sólo del porcentaje de heterocromatina o si las secuencias que conforman los knobs podrían explicar dichas relaciones.

Kato et al. (2009) consideran que los knobs deben estar sujetos a la acción de la selección tanto natural como artificial y, por tanto, adquieren valores adaptativos variables y se convierten en elementos muy importantes en la evolución de las poblaciones de Zea.

A nivel molecular, los knobs están constituidos por una familia de secuencias de 180 pares de bases (180-pb) y otra de 350 pares de bases (TR-1), y pueden albergar varios retrotransposones (Peacock et al., 1981; Dennis y Peacock, 1984; Ananiev et al., 1998a y b). Ambas familias están representadas por miles a millones de copias dispuestas en tándem, en distintas proporciones unas de otras, y constituyen los tres tipos de knobs: los formados exclusivamente por repeticiones de 180-pb, los formados exclusivamente por repeticiones de TR-1, y los formados por ambas secuencias en distintas proporciones (Ananiev et al., 1998a y b). Estos autores encontraron en la secuencia TR-1 dos regiones, una de $31 \mathrm{pb}$ y otra de $12 \mathrm{pb}$, que son homólogas a la secuencia 180 -pb, por lo que propusieron que TR-1 habría evolucionado a partir de la secuencia 180 -pb por duplicación y posterior divergencia.
Si bien el número y posición de los knobs han sido utilizados por muchos autores para la caracterización de razas de maíz de Latinoamérica (Longley, 1938; Roberts et al., 1957; Wellhausen et al., 1957; Ramírez et al., 1960; Grobman et al., 1961; Kato, 1976; McClintock et al., 1981), no se registran datos de caracterizaciones citogenéticas que tengan en cuenta la composición de secuencias de los knobs, tanto en maíz como en teocintle. La técnica de hibridación in situ fluorescente (FISH) permite la detección y localización de secuencias específicas sobre núcleos interfásicos y cromosomas. Con el uso de las secuencias $180-$ pb y TR-1 como sondas en experimentos de FISH es posible revelar la composición molecular de los knobs (Albert et al., 2010; González y Poggio 2011).

$\mathrm{Al}$ aplicar técnicas de citogenética clásica (tinción DAPI) y molecular (FISH) en este trabajo se demuestra que, además del número y tamaño de los knobs, su composición de secuencias es de gran utilidad en la caracterización de distintas accesiones de maíz y teocintle.

\section{MATERIALES Y MÉTODOS}

Los materiales utilizados para este trabajo, con sus accesiones y procedencias, se listan en el Cuadro 1. Las razas de maíz empleadas son nativas del Noroeste de Argentina (NOA) y provienen de poblaciones originales cultivadas a diferentes altitudes en las Provincias de Tucumán, Salta y Jujuy. Las mismas fueron cedidas por Ing. Agr. Julián Cámara Hernández del Laboratorio Vavilov de la Facultad de Agronomía (FA) de la Universidad de Buenos Aires (UBA), y por el banco de semillas de INTA-Pergamino (Instituto Nacional de Tecnología Agropecuaria). Los teocintles fueron cultivados y mantenidos en el campo experimental de la FA-UBA. Se analizaron entre cuatro y siete individuos de cada accesión y al menos 10 células por individuo.

\section{Preparaciones cromosómicas}

Para su germinación las semillas fueron colocadas en cajas de Petri con papel de filtro embebido en agua estéril. Las raíces fueron pretratadas en una solución de 8-hidroxiquinoleína $(0.002 \mathrm{M})$ durante $3 \mathrm{~h}$ a $20^{\circ} \mathrm{C}$ y fijadas en una solución de alcohol etílico absoluto-ácido acético (3:1). Los ápices radicales se lavaron en amortiguador (ácido cítricocitrato de sodio $0.01 \mathrm{M}, \mathrm{pH} 4.6$ ) antes y después de ser tratados con una solución enzimática con $2 \%$ de celulasa (Onozuka R10, Merck ${ }^{\circledR}$ ) y $20 \%$ de pectinasa (SIGMA ${ }^{\circledR}$ P4716) durante $30 \mathrm{~min}$ a $37^{\circ} \mathrm{C}$. Finalmente, se realizó el aplastado del tejido sobre una gota de ácido acético (45\%) en un portaobjetos. Luego de remover el cubreobjetos por congelamiento, las preparaciones fueron secadas al aire para su utilización en los experimentos de FISH. 
Cuadro 1. Accesiones de maíz y de teocintle con sus respectivas altitudes de cultivo, localidades de colección y orígenes.

\begin{tabular}{|c|c|c|c|}
\hline $\begin{array}{l}\text { Materiales de } Z e a \\
\text { (altitud de cultivo) }\end{array}$ & Accesión & Localidad & Legado por \\
\hline $\begin{array}{l}\text { Maíz Raza Capia Blanco } \\
(2800 \mathrm{~m})\end{array}$ & ARZM09291 & Uquía, Depto. de Humahuaca, Jujuy, Argentina & INTA Pergamino \\
\hline $\begin{array}{l}\text { Maíz Raza Amarillo Chico } \\
(2000 \mathrm{~m})\end{array}$ & VAV 6451 & $\begin{array}{l}\text { El Condado, Depto. de Santa Victoria, } \\
\text { Salta, Argentina }\end{array}$ & $\begin{array}{l}\text { Lab. Vavilov } \\
\text { (FA- UBA) }\end{array}$ \\
\hline $\begin{array}{l}\text { Maíz Raza Amarillo Grande } \\
(2000 \mathrm{~m})\end{array}$ & VAV 6669 & $\begin{array}{l}\text { Molulo, Depto. Dr. Manuel Belgrano, } \\
\text { Jujuy, Argentina }\end{array}$ & $\begin{array}{l}\text { Lab. Vavilov } \\
\text { (FA-UBA) }\end{array}$ \\
\hline $\begin{array}{l}\text { Maíz Raza Amarillo Chico } \\
(1690 \mathrm{~m})\end{array}$ & VAV 6476 & $\begin{array}{l}\text { Termas de Reyes, Depto. Dr. Manuel Belgrano, } \\
\text { Jujuy, Argentina }\end{array}$ & $\begin{array}{l}\text { Lab. Vavilov } \\
\text { (FA- UBA) }\end{array}$ \\
\hline $\begin{array}{l}\text { Maíz Raza Blanco y Ocho Rayas } \\
(1250 \mathrm{~m})\end{array}$ & VAV 6483 & $\begin{array}{l}\text { Las Arcas, Depto. de Trancas, Tucumán, } \\
\text { Argentina }\end{array}$ & $\begin{array}{l}\text { Lab. Vavilov } \\
\text { (FA- UBA) }\end{array}$ \\
\hline $\begin{array}{l}\text { Maíz Raza Calchaquí } \\
(1120 \mathrm{~m})\end{array}$ & ARZM08068 & $\begin{array}{l}\text { Buena Vista, Depto. de Guachipas, Salta, } \\
\text { Argentina }\end{array}$ & $\begin{array}{l}\text { INTA } \\
\text { Pergamino }\end{array}$ \\
\hline $\begin{array}{l}\text { Maíz Raza Orgullo Cuarentón } \\
(910 \mathrm{~m})\end{array}$ & VAV 6482 & $\begin{array}{l}\text { La Candelaria, } \\
\text { Depto. de La Candelaria, Salta, Argentina. }\end{array}$ & $\begin{array}{l}\text { Lab. Vavilov } \\
\text { (FA-UBA) }\end{array}$ \\
\hline Maíz Línea (0 m) & 13043 & $\begin{array}{l}\text { Ciudad Autónoma de Buenos Aires, } \\
\text { Argentina }\end{array}$ & FA-UBA \\
\hline Zea luxurians (900 m) & cv. 2228 & Guatemala & Dra. C. Prywer \\
\hline Zea diploperennis (1860 m) & cv. 2232 & San Miguel, Ciudad Guzmán, Jalisco, México & Dra. C. Prywer \\
\hline $\begin{array}{l}\text { Zea mays ssp. parviglumis } \\
(590 \mathrm{~m})\end{array}$ & cv. 8391 & Valle del Río Balsas, Guerrero, México & CIMMYT \\
\hline $\begin{array}{l}\text { Zea mays ssp. mexicana } \\
(1860 \mathrm{~m})\end{array}$ & KG-04-2 & Chalco-Amecameca, Mesa Central, México & $\begin{array}{l}\text { Dr. A. T. Kato } \\
\text { Yamakake }\end{array}$ \\
\hline $\begin{array}{l}\text { Zea perennis } \\
(2120 \mathrm{~m})\end{array}$ & cv. 2257 & $\begin{array}{l}\text { Piedra Ancha, San Gabriel, } \\
\text { Jalisco, México }\end{array}$ & Dra. C. Prywer \\
\hline
\end{tabular}

FA-UBA = Facultad de Agronomía de la Universidad de Buenos Aires; INTA = Instituto Nacional de Tecnología Agropecuaria; CIMMYT = Centro Internacional de Mejoramiento de Maíz y Trigo.

\section{Obtención y marcado de las sondas knobs}

Las secuencias repetitivas 180-pb y TR-1 (Peacock et al., 1981; Ananiev et al., 1998a) de los segmentos heterocromáticos del knob de maíz fueron obtenidas del banco de datos GenBank (www.ncbi.nlm.nih.gov/genbank/). Con el programa "Primer3" 0.6 (Rozen y Skaletsky, 2000) se diseñaron los cebadores para amplificar las dos secuencias componentes de los knobs. Luego se ordenó la síntesis de estos cebadores, cuyas secuencias fueron: para 180-pb (S: 3' ATAGCCATGAACGACCAT 5' y A: 5' ACCCCACATATGTTTCCT 3'), y para TR-1 (S: 3' TCAACTTTTAAACCACAAATGATG 5' y A: 5' GTTTGGaCAGTTCACTCATGCA 3'), en Promega ${ }^{\circledR}$.

Para hacer las mezclas de amplificación se colocaron 5 $\mu \mathrm{L}$ de una dilución de $\mathrm{ADN}$ genómico de maíz de la raza Amarillo Chico (50 a $100 \mathrm{ng}$ ), $10 \mu \mathrm{L}$ de una mezcla de nucleótidos (dNTPs) $(200 \mu \mathrm{M}$ de cada dNTP), $3 \mu \mathrm{L}$ de ClMg (2.5 mM), $0.25 \mu \mathrm{L}$ de enzima Taq polimerasa (1 unidad), 3 $\mu \mathrm{L}$ de amortiguador $10 \mathrm{X}$ de Taq, $5 \mu \mathrm{L}$ de una dilución de cada cebador ( $1 \mu \mathrm{M}$ de cada uno) y agua destilada estéril hasta un volumen de $30 \mu \mathrm{L}$.

La amplificación se hizo en un termociclador con el siguiente programa: 1 ciclo de 4 min a $94{ }^{\circ} \mathrm{C}$, 35 ciclos de 1 min a $94{ }^{\circ} \mathrm{C}, 1 \min$ a $47^{\circ} \mathrm{C}$ y $1 \min$ a $72^{\circ} \mathrm{C}$, y 1 ciclo de 1 min a $72{ }^{\circ} \mathrm{C}$. Los productos de amplificación se separaron por electroforesis en un gel $0.8 \%$ de agarosa. Las secuencias amplificadas se extrajeron del gel con QIAquick Gel Extraction Kit (QIAGEN®) según las instrucciones del fabricante. El marcado de las sondas se hizo con Dig-High Prime y Biotin-Nick Translation Mix (Roche $\left.{ }^{\circledR}\right)$, según las instrucciones de los fabricantes.

\section{Hibridación in situ fluorescente (FISH)}

La técnica se aplicó según González et al. (2004). Los preparados se pretrataron con una dilución 1:100 de RNAsa $\left(10 \mathrm{mg} \mathrm{mL}^{-1}\right)$ en el amortiguador de citrato sodico-ClNa 
2XSSC (Saline-Sodium Citrate) por $1 \mathrm{~h}$, se incubaron en pformaldehído $4 \%$ (p/v) por $10 \mathrm{~min}$, se lavaron tres veces en $2 \mathrm{x}$ SSC por 5 min cada vez, se deshidrataron en una serie alcohólica de etanol $\left(70^{\circ}, 90^{\circ}\right.$ y $100^{\circ}$ durante 3 min por cada graduación) y se secaron al aire.

Para la mezcla de hibridación se colocaron: $15 \mu \mathrm{L}$ de formamida pura, $6 \mu \mathrm{L}$ de sulfato de dextrano, $3 \mu \mathrm{L}$ de amortiguador 20XSSC, $1 \mu \mathrm{L}$ de SDS $10 \%, 1 \mu \mathrm{L}$ de ADN de esperma de salmón y 100 a 200 ng de la secuencia knob marcada. Se añadieron $30 \mu \mathrm{L}$ de la mezcla de hibridación a cada una de las preparaciones. Las mismas se colocaron en un termociclador con el siguiente programa: $7 \mathrm{~min}$ a $75^{\circ} \mathrm{C}, 30 \mathrm{~s}$ a 55 ${ }^{\circ} \mathrm{C}, 1 \mathrm{~min}$ a $45^{\circ} \mathrm{C}, 2 \mathrm{~min}$ a $42^{\circ} \mathrm{C}, 5 \mathrm{~min}$ a $40^{\circ} \mathrm{C}$ y 5 min a 38 ${ }^{\circ} \mathrm{C}$. Después se incubaron en estufa a $37^{\circ} \mathrm{C}$ en una cámara húmeda, durante $12 \mathrm{~h}$.

Los lavados posteriores a la hibridación se efectuaron con agitación y en condiciones de alta astringencia, en las cuales sólo permanecen hibridadas las secuencias que superan el $85 \%$ de homología entre sí. Las condiciones de lavado fueron los siguientes: 2 x SSC a $42^{\circ} \mathrm{C}$ por $5 \mathrm{~min}$, formamida deionizada a $20 \%$ (v/v) en $0.1 \times$ SSC a $42{ }^{\circ} \mathrm{C}$ por $10 \mathrm{~min}, 0.1$ x SSC a $42{ }^{\circ} \mathrm{C}$ durante $5 \mathrm{~min}, 4 \mathrm{x} \mathrm{SSC} /$ Tween $(0.2 \%)$ a $42{ }^{\circ} \mathrm{C}$ por $5 \mathrm{~min}, \mathrm{y} 4 \mathrm{x} \mathrm{SSC} /$ Tween $(0.2 \%)$ a $20^{\circ} \mathrm{C}$ durante $5 \mathrm{~min}$.

Sobre cada preparación se colocaron 100 uL de amortiguador de detección: BSA (albúmina bovina) a $5 \%(\mathrm{p} / \mathrm{v})$ en $4 \mathrm{x} \mathrm{SSC} /$ Tween 20 , y se incubaron a $25^{\circ} \mathrm{C}$ por $5 \mathrm{~min}$. La detección se realizó al agregar a cada preparación $50 \mu \mathrm{L}$ de una solución a $2 \%(\mathrm{v} / \mathrm{v})$ de antidigoxigenina-FITC en BSA $5 \%$ y Streptavidina-Cy3 $0.2 \%$ (v/v) en BSA $5 \%$, para detectar las sondas marcadas con digoxigenina y biotina respectivamente. Las preparaciones se incubaron a $37^{\circ} \mathrm{C}$ en cámara húmeda y en oscuridad por $1 \mathrm{~h}$ y se lavaron tres veces en $4 \times$ SSC/Tween $(0.2 \%)$ a temperatura ambiente y en oscuridad durante $10 \mathrm{~min}$. Finalmente se tiñeron con $100 \mu \mathrm{L}$ de 4', 6'-diamino-2-fenilindol (DAPI) $\left(1 \mu \mathrm{g} \mathrm{mL}^{-1}\right.$ en amortiguador citrato McIlvaine $\mathrm{pH}$ 7) por $30 \mathrm{~min}$, se lavaron rápidamente en $4 \mathrm{x}$ SSC y se montaron con una gota de Vectashield ${ }^{\circledR}$.

Las preparaciones se examinaron con microscopio de epifluorescencia (Axiophot, Carl Zeiss ${ }^{\circledR}$ ) y las microfotografías se tomaron con una cámara digital (CCD, Leica $\left.{ }^{\circledR}\right)$. Las imágenes fueron integradas con el programa Adobe Photoshop CS2 v 9.0 (Adobe Systems Incorporated ${ }^{\circledR}$, USA).

\section{RESULTADOS}

En núcleos interfásicos de diferentes accesiones de maíz y teocintle se evidenció el número de regiones DAPI+ mediante tinción con DAPI, mientras que en metafases mitóticas con esta tinción se observó el número de bandas
DAPI+. En los casos en los que se analizaron metafases mitóticas se observó que en los distintos maíces la posición cromosómica de las bandas DAPI+ es principalmente subterminal al igual que en $Z$. $m$. ssp. parviglumis y $Z$. $m$. ssp. mexicana, mientras que en $Z$. luxurians y en $Z$. diploperennis es terminal (Figura 1, G-K).

El FISH con el uso simultáneo de las secuencias knobs 180-pb y TR-1 como sondas, reveló la constitución de secuencias en cada región/banda DAPI+ (Figura 1 y Cuadro 2). Se observaron knobs (regiones/bandas DAPI+) compuestos exclusivamente por la secuencia 180-pb, otros formados solamente por la secuencia TR-1, y knobs constituidos por ambas secuencias, las que mostraron estar en yuxtaposición y tener diferente proporciones relativas en la constitución de diferentes knobs. En el Cuadro 2 se muestra el patrón observado para cada accesión en cuanto al número y constitución de secuencia de sus knobs.

En núcleos y metafases del maíz de la raza Blanco y Ocho Rayas (accesión: VAV 6483) se observaron 13 regiones DAPI+ (Figura 1, A). Todas estas regiones hibridaron con la secuencia 180-pb marcada con biotina (Figura 1, B), de las cuales sólo tres presentaron además señal de hibridación positiva con la secuencia TR-1 marcada con digoxigenina (Figura 1, C).

En la raza de maíz Amarillo Chico (accesión: VAV 6451), la secuencia TR-1 hibridó en forma exclusiva sobre dos de las 15 regiones DAPI+ observadas, mientras que la secuencia 180-pb hibridó en 11 de estas regiones y sólo dos mostraron señal de hibridación con ambas secuencias. En otra población de la misma raza (accesión: VAV 6476), colectada en otra localidad a diferente altitud, se observaron diferencias con respecto a la población anterior. Amarillo Chico (accesión: VAV 6476) presentó 12 bandas DAPI+, de las cuales seis hibridaron exclusivamente con la secuencia $180-$ pb y las otras seis mostraron señales de hibridación con ambas secuencias knob.

Al hibridar núcleos interfásicos del maíz Orgullo Cuarentón (accesión: VAV 6482) se observaron 15 regiones DAPI+ (Figura 1, D), de las cuales dos presentaron señales de hibridación positiva sólo con la secuencia TR-1 marcada con biotina (Figura 1, E), cinco con la secuencia 180-pb marcada con digoxigenina (Figura 1, F), y otras ocho mostraron señales de hibridación con ambas secuencias (Figura 1, E y F).

En la raza de maíz Calchaquí (accesión: ARZM08068) los núcleos interfásicos mostraron ocho regiones DAPI+, de las cuales cuatro hibridaron exclusivamente con la secuencia $180-$ pb y las cuatro restantes con ambas secuencias knob. La intensidad de hibridación de la secuencia 

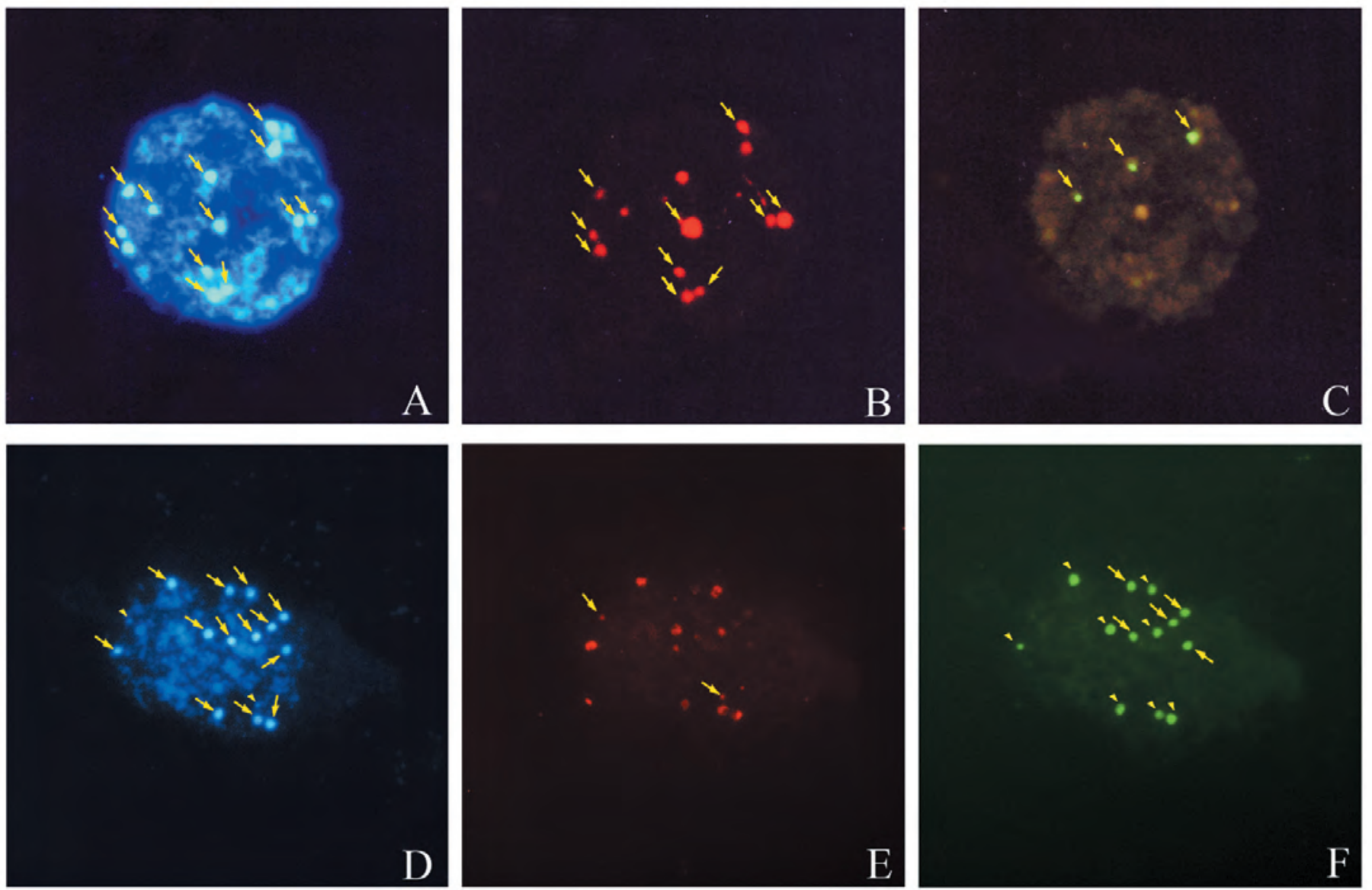

Figura 1 (A-F). A-C: Núcleo interfásico de la raza de maíz Blanco y Ocho Rayas (accesión: VAV6483). A: tinción DAPI (azul); las flechas indican las regiones DAPI+. B: FISH con sonda de secuencia 180-pb marcada con biotina y detectada con Cy3 (rojo); las flechas señalan los knobs hibridados exclusivamente con la secuencia 180-pb. C: FISH con sonda de secuencia TR-1 marcada con digoxigenina y revelada con antidigoxigenin-FITC; las flechas muestran los knobs hibridados positivamente (verde). D-F: Núcleo interfásico de la raza de maíz Orgullo Cuarentón (accesión VAV6482). D: tinción DAPI; las flechas señalan 13 regiones DAPI+ conspicuas y las puntas de flecha muestran dos señales DAPI+ débiles. E: FISH con sonda de secuencia TR-1 marcada con biotina y detectada con Cy3 (rojo); las flechas indican los knobs hibridados exclusivamente con la secuencia TR-1. F: FISH con sonda de secuencia 180-pb marcada con digoxigenina y revelada con antidigoxigenin-FITC; las flechas muestran los knobs hibridados exclusivamente con las secuencia 180-pb, y las puntas de flechas indican el resto de los knobs que fueron hibridados con dicha secuencia (verde).

TR-1 sobre una de estas últimas cuatro regiones fue menor que la intensidad de hibridación de la secuencia 180pb. Las interfases del maíz raza Capia Blanco (accesión: ARZM09291) también presentaron ocho regiones DAPI+, pero sólo dos de ellas hibridaron únicamente con la secuencia 180-pb, otras dos hibridaron sólo con la secuencia TR-1 y las cuatro restantes con ambas secuencias. Las señales de hibridación con la secuencia 180 -pb resultaron ser de mayor tamaño y más intensas que las señales de hibridación de la secuencia TR-1.
En los experimentos de FISH hechos sobre núcleos interfásicos y metafases mitóticas del maíz de la raza Amarillo Grande (accesión: VAV 6669) se observaron 11 bandas DAPI+, de las cuales dos mostraron señal de hibridación exclusivamente con la secuencia 180-pb (marcada con digoxigenina), una hibridó únicamente con la secuencia TR-1 (marcada con biotina) y las ocho restantes mostraron señales de hibridación con ambas secuencias. Dos de estas últimas se localizaron sobre los satélites que se encuentran en el par cromosómico 6 (Figura 1, G). 

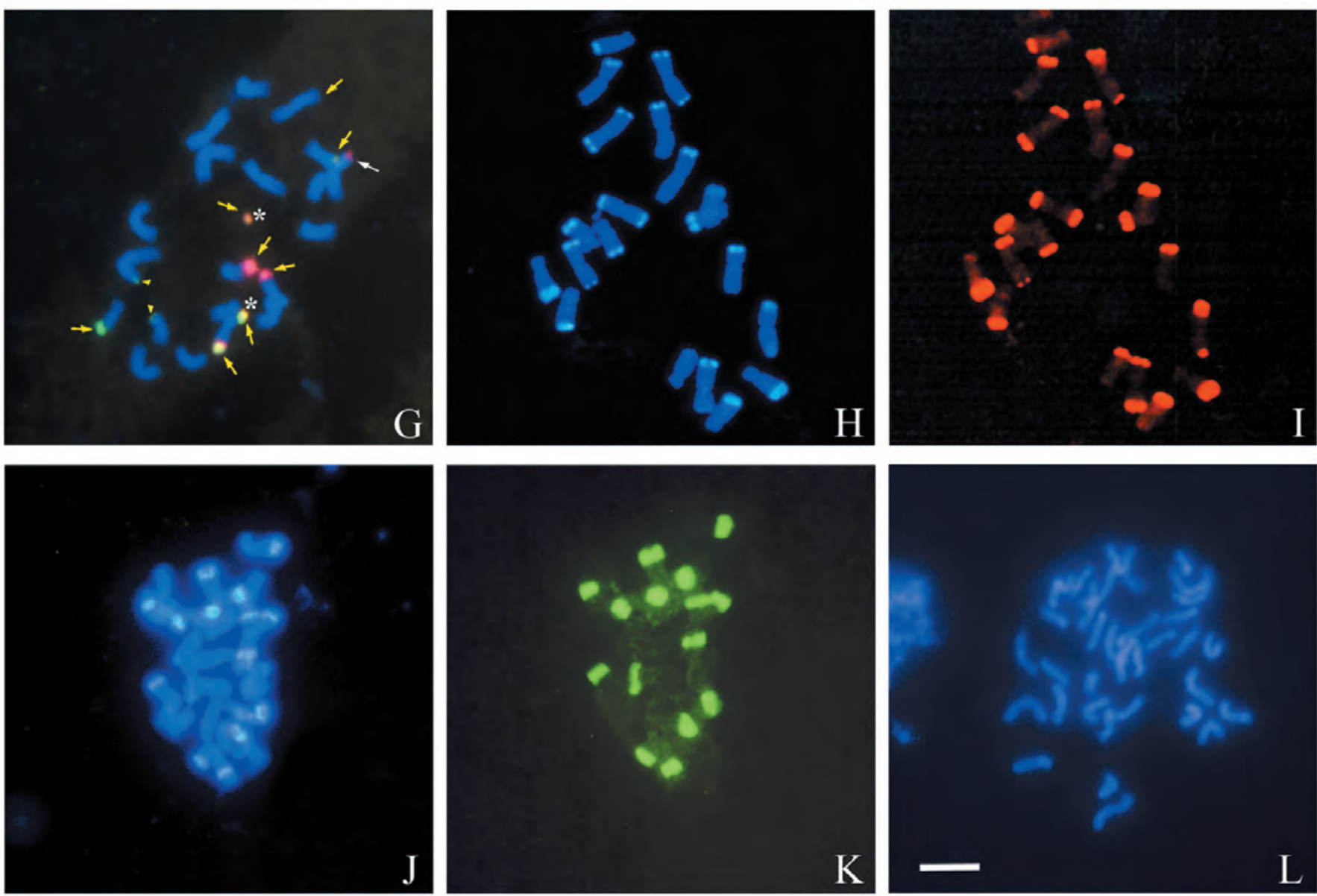

Figura 1 (Continuación G-L). G: Metafase mitótica de la raza de maíz Amarillo Grande (accesión: VAV 6669) teñida con DAPI (azul) e hibridada con sonda de secuencia TR-1 marcada con biotina (detectada con Cy3, rojo) y con sonda de secuencia 180-pb marcada con digoxigenina (revelada con antidigoxigenin-FITC, verde); los asteriscos marcan el par de satélites, las flechas amarillas muestran los knobs hibridados con ambas secuencias, las puntas de flecha señalan los knobs hibridados exclusivamente con la secuencia 180-pb, y la flecha blanca indica el knob que hibridó sólo con la secuencia TR-1. H-I: Metafase mitótica de Zea luxurians. H: Tinción con DAPI (azul). I: FISH con sonda de secuencia 180-pb marcada con biotina y detectada con Cy3 (rojo). J-K: Metafase mitótica de Zea mays ssp. parviglumis. J: tinción con DAPI (azul). K: FISH con sonda de secuencia 180-pb marcada con digoxigenina y revelada con antidigoxigenin-FITC (verde). L: Tinción con DAPI de metafase mitótica de Zea perennis (azul). Barra: $10 \mu \mathrm{m}$.

Las metafases mitóticas de la línea de maíz 13043 mostraron 10 bandas DAPI+, las cuales hibridaron positivamente con la secuencia 180 -pb. Únicamente cuatro de ellas mostraron señales de hibridación con la secuencia TR-1. Las mismas fueron de menor tamaño e intensidad que las de la secuencia 180-pb. Además, dos de estos knobs, compuestos por ambas secuencias, fueron localizados sobre los satélites del par cromosómico 6.

En cuanto a los resultados obtenidos en los teocintles, la tinción DAPI reveló que $Z$. luxurians posee la mayor cantidad de bandas DAPI+ (26 a 28) y que son de mayor tamaño que en maíz y en el resto de los teocintles (Figura 1, H). Por su parte, Z. m. ssp. parviglumis mostró 14 a 15 bandas DAPI+ (Figura 1, J), Z. m. ssp. mexicana exhibió ocho re- giones DAPI+, Z. diploperennis mostró 16 bandas DAPI+, y Z. perennis no presentó estas bandas (Figura 1, L).

Al aplicar el método FISH sobre metafases mitóticas de Z. luxurians y Z. m. ssp. parviglumis, con el empleo de la secuencia knob 180-pb como sonda, se observaron fuertes señales de hibridación sobre todas las bandas DAPI + (Figura $1, \mathrm{H}-\mathrm{K}$ ). El mismo experimento realizado sobre Z. $m$. ssp. mexicana reveló que de sus ocho regiones DAPI+ dos no presentaron señales de hibridación y las seis restantes mostraron señales de hibridación de baja intensidad.

Los experimentos de FISH en los que se hibridaron simultáneamente las secuencias 180-pb (marcada con digoxigenina) y TR-1 (marcada con biotina) sobre metafases 
Cuadro 2. Número de bandas/regiones DAPI+, número de knobs compuestos exclusivamente por la secuencia 180-pb, número de knobs constituidos únicamente por la secuencia TR-1, y número de knobs compuestos por ambas secuencias, en cada accesión.

\begin{tabular}{|c|c|c|c|c|}
\hline Materiales de Zea (altitud de cultivo) & Bandas DAPI+ & $180-\mathrm{pb}$ & TR-1 & $180 \mathrm{pb}+\mathrm{TR}-1$ \\
\hline Maíz Raza Amarillo Chico (VAV6451) (2000 m) & 15 & 11 & 2 & 2 \\
\hline Maíz Raza Orgullo Cuarentón (VAV6482) (910 m) & 15 & 5 & 2 & 8 \\
\hline Maíz Raza Amarillo Chico (VAV6476) (1690 m) & 12 & 6 & 0 & 6 \\
\hline Maíz Raza Blanco y Ocho Rayas (VAV6483) (1250 m) & 13 & 10 & 0 & 3 \\
\hline Maíz Raza Amarillo Grande (VAV6669) (2000 m) & 11 & 2 & 1 & 8 \\
\hline Maíz Raza Calchaquí (1120 m) & 8 & 4 & 0 & 4 \\
\hline Maíz Raza Capia Blanco (2800 m) & 8 & 2 & 2 & 4 \\
\hline Maíz Línea 13043 (0 m) & 10 & 6 & 0 & 4 \\
\hline Zea luxurians cv. 2228 (900 m) & $26-28$ & $26-28$ & 0 & $\mathrm{~s} / \mathrm{d}$ \\
\hline Zea mays ssp. mexicana Mesa Central KG-04-2 (1860 m) & 8 & 6 & $s / d$ & $s / d$ \\
\hline Zea mays ssp. parviglumis Balsas cv. 8391 (590 m) & $14-15$ & $14-15$ & 0 & 0 \\
\hline Zea diploperennis San Miguel cv. 2232 (1860 m) & 16 & 0 & 0 & 16 \\
\hline Zea perennis Piedra Ancha cv. 2257 (2120 m) & 0 & 0 & 0 & 0 \\
\hline
\end{tabular}

mitóticas de Z. diploperennis y de Z. perennis, en la primera especie mostraron señales de hibridación de similar intensidad con ambas secuencias en todas las bandas DAPI+; mientras que en la segunda especie los cromosomas no presentaron bandas DAPI+ ni señales de hibridación (Figura $1, \mathrm{~L})$.

\section{DISCUSIÓN}

En Zea la heterocromatina forma bloques citológicamente observables denominados nudos cromosómicos (knobs, en inglés) (Kato, 1976; McClintock, 1978). Estos bloques se encuentran principalmente en posición subterminal en el maíz, en Z. m. ssp. mexicana y Z. m. ssp. parviglumis, y terminal en $Z$. luxurians y en $Z$. diploperennis (Kato, 1976; Kato y López, 1990). En este trabajo se analizó si las variaciones en la cantidad de regiones knob y en la composición de sus secuencias permiten una caracterización citogenética más completa de accesiones del género Zea. Para ello se utilizó una combinación de técnicas de citogenética clásica (tinción DAPI) y molecular (FISH).

La tinción con el fluorocromo DAPI, que identifica a los knobs como bandas/regiones DAPI+ (Poggio et al., 1998; González y Poggio, 2011), mostró que su número es variable entre las accesiones analizadas. En los experimentos de FISH realizados en este trabajo, en los que se hibridaron simultáneamente las secuencias knob 180-pb y TR-1 sobre metafases o núcleos interfásicos de las accesiones estudiadas, se observó una co-localización de las regiones DAPI+ con las señales de hibridación. Estos experimentos permi- tieron detectar la o las secuencias componentes de cada región/banda DAPI+. Ambas secuencias se encontraron, combinadas o aisladas, en los distintos knobs.

Dentro de cada accesión de maíz estudiada se encontró constancia en el número y composición de secuencias $k n$ $o b s$, pero esta característica fueron variables entre accesiones. En los individuos de la raza Amarillo Chico (accesión: VAV 6451) se observaron 15 regiones DAPI+ correspondientes a los knobs, que confirman los resultados de Poggio et al. (1998) y más recientemente de González y Poggio (2011).

En el presente trabajo se reveló además, la composición de secuencia de cada uno de estos knobs. En la raza Amarillo Chico (accesión VAV 6451) la tinción DAPI reveló 15 knobs al igual que en la raza Orgullo Cuarentón (accesión VAV 6482), pero la composición de secuencia de los mismos varió entre ambas accesiones. También, el mismo número de knobs pero con variación en la composición de secuencias de los mismos fue detectada entre las razas Calchaquí (accesión ARZM08068) y Capia Blanco (accesión ARZM09291). Estos resultados demuestran la utilidad de las técnicas de FISH en la diferenciación citogenética de accesiones de maíz.

Con los resultados obtenidos en maíz se observó que a menor altitud de cultivo los knobs estaban compuestos solamente por la secuencia 180-pb o en combinación con la secuencia TR-1, a excepción del maíz Orgullo Cuarentón que posee un par de knobs compuestos sólo por la secuencia 
TR-1. La presencia de knobs constituidos exclusivamente por la secuencia TR-1 se observó solo en los maíces provenientes de mayores altitudes de cultivo. Estos datos plantean el interrogante de si la composición de los knobs estaría relacionada con las correlaciones inversas observadas por Rosato et al. (1998) y Poggio et al. (1998) entre el porcentaje de heterocromatina y la altitud de cultivo, o entre el porcentaje de heterocromatina y el número de cromosomas B. Para dilucidar este interrogante es necesario ampliar el número de accesiones a analizar, y estudiar en detalle el patrón de knobs, porcentaje de hetrocromatina y número de cromosomas B en las razas de una región a distintas altitudes de cultivo, por ejemplo en el Noroeste de Argentina. La asociación entre número y constitución de secuencias de los knobs en relación con la altitud de cultivo, la convierte en un interesante modelo para el análisis del valor selectivo de estas regiones heterocromáticas.

En este trabajo se observó, mediante FISH con las secuencias knobs, que las regiones DAPI+ de mayor tamaño presentan señales de hibridación mayores y más intensas. Ananiev et al. (1998a), al hibridar cromosomas de maíz con la secuencia $180-\mathrm{pb}$, postularon una correlación positiva entre el tamaño de los knobs y la cantidad de repeticiones que los componen. Esto demuestra que los knobs que mostraron mayores y más intensas señales de hibridación están compuestos por un mayor número de repeticiones en tandem de las secuencias con las que fueron hibridadas.

En lo que respecta a teocintles, Z. m. ssp. parviglumis, considerado por varios autores como el antecesor del maíz (Buckler y Holstford, 1996; Matsuoka et al., 2002), presentó un número elevado de knobs conspicuos. Todos ellos hibridaron intensamente con la secuencia 180-pb. Al hibridar la misma secuencia sobre núcleos interfásicos de otra accesión de Z. m. ssp. parviglumis, Poggio et al. (2005) observaron el mismo número de regiones DAPI+ pero algunas de ellas no tuvieron homología con la secuencia 180-pb. Esto muestra la variación que puede encontrarse entre accesiones de diferentes localidades.

En la accesión de Z. m. ssp. mexicana se observaron ocho knobs, y sólo seis estaban compuestos por la secuencia 180pb. Z. luxurians mostró el mayor número y tamaño de knobs que en el resto de especies Zea. Los mismos se encontraron en posición terminal y en ambos brazos de la mayoría de los cromosomas. Todos estos knobs mostraron estar compuestos por la secuencia $180-\mathrm{pb}$ puesto que presentaron fuertes señales de hibridación sobre todas las bandas DAPI+. Estos experimentos evidencian que $Z$. luxurians no posee knobs compuestos exclusivamente de TR-1. Estos resultados coinciden con los descritos recientemente por Albert et al. (2010) y González y Poggio (2011), y ponen de manifiesto que la secuencia TR-1 se encuentra poco representada en el genoma de Z. luxurians. En Z. diploperennis se observaron proporciones similares de ambas secuencias en todos sus knobs, en coincidencia con los resultados de Albert et al. (2010).

En los experimentos de FISH Z. perennis no mostró bandas DAPI+ ni señales de hibridación con las secuencias $180-\mathrm{pb}$ y TR-1. Z. perennis es la especie de Zea que posee el menor contenido de ADN por genoma básico (Tito et al., 1991). Tampoco González et al. (2006) observaron señales de hibridación sobre los knobs del maíz al hibridar ADN genómico de esta especie sobre metafases mitóticas de maíz. Por tanto, nuestros resultados confirman la ausencia de estas secuencias en el genoma de $Z$. perennis, y sugieren que la disminución del contenido de ADN en el genoma básico de esta especie consistió principalmente en la pérdida de las secuencias repetitivas que constituyen los knobs. Esta pérdida sería una consecuencia del proceso de poliploidización secundaria de esta especie (Poggio et al., 2005). Por el contrario, Zea luxurians posee el mayor contenido de ADN del género (Tito et al., 1991) que podría explicarse por el mayor número y tamaño de knobs observados en esta especie.

El análisis conjunto de los resultados de las accesiones de maíz analizadas mostró que la mayor parte de los knobs estaban compuestos exclusivamente por la secuencia $180-\mathrm{pb}$, mientras que un número mucho menor solamente por TR1. Asimismo, en los teocintles se detectó, con excepción de $Z$. diploperennis, una predominancia de knobs conformados por la secuencia $180-\mathrm{pb}$. La preponderancia de la secuencia de 180-pb podría deberse a que los elementos TR-1 evolucionaron a partir de repeticiones de 180-pb como resultado de una duplicación y subsecuente divergencia (Ananiev et al., 1998b).

Los resultados de los experimentos de FISH aquí realizados sobre las metafases mitóticas indican que es posible elaborar cariotipos FISH propios de distintas accesiones de maíces y teocintles, lo que permitirá una caracterización citogenética más completa de Zea. Estas metodologías pueden extrapolarse también a la caracterización y reconocimiento de líneas e híbridos comerciales de maíz, como quedó demostrado con los resultados obtenidos para la línea 13043 aquí estudiada.

\section{CONCLUSIONES}

Las tinciones DAPI revelaron la existencia de variación en bandas/regiones heterocromáticas (knobs) sobre los cromosomas o núcleos interfásicos respectivamente, variación que es de utilidad para la caracterización de accesiones de Zea. Los resultados de FISH demostraron variación entre accesiones en el tipo de secuencias que componen los diferentes knobs. Con los patrones de hibridación diferenciales obtenidos fue posible discernir entre las accesiones tanto de 
maíz como de teocintle aquí estudiadas, aportar datos para la caracterización citogenética y contribuir al conocimiento de la variabilidad genética del género.

La caracterización citogenética de maíces y teocintles mediante el estudio de sus knobs, es un procedimiento rápido y factible que vislumbra nuevas perspectivas para profundizar estudios biosistemáticos y establecer hipótesis acerca del origen y el valor adaptativo de las regiones heterocromáticas. Esta caracterización es un aporte al conocimiento de la variabilidad fenotípica y genotípica de los taxa del género Zea, por lo cual sería de utilidad e importancia su integración en programas de mejoramiento y de conservación de la biodiversidad.

\section{AGRADECIMIENTOS}

Al Ing. Agr. Julián Cámara-Hernández por proveer las accesiones analizadas de maíz y al Sr. Diego Fink por la asistencia técnica con el material fotográfico. A la Agencia Nacional de Promoción Científica y Técnica-FONCYT (PICT-14119), a la Universidad de Buenos Aires (UBACYT: $\mathrm{X}-178$ ) y al CONICET (PIP-0342) por el financiamiento de este trabajo.

\section{BIBLIOGRAFÍA}

Albert P S, Z Gao, T V Danilova, J A Birchler (2010) Diversity of chromosomal karyotypes in maize and its relatives. Cytogen. Genome Res. 129:6-16.

Ananiev E V, R L Phillips, H W Rines (1998a) A knob associated tandem repeat in maize capable of forming fold-back DNA segments: are chromosome knobs megatransposons? PNAS USA-Biol. Sci. 95:10785-10790.

Ananiev E V, R L Phillips, H W Rines (1998b) Complex structure knob DNA of maize chromosome 9: Retrotransposon invasion into heterochromatin. Genetics 149:2025-2037.

Buckler E S IV, T P Holtsford (1996) Zea systematics: Ribosomal ITS evidence. Mol. Biol. Evol. 13:612-622.

Cámara H J, D Arancibia de C (2007) Maíces Andinos y sus Usos en la Quebrada de Humahuaca y Regiones Vecinas. 1a ed. Facultad de Agronomía-Universidad de Buenos Aires, Argentina. 60 p.

Dennis E, W Peackock (1984) Knob heterochromatin homology in maize and its relatives. J. Mol. Biol. 20:341-350.

GenBank. National Center for Biotechnology Information. http://www. ncbi.nlm.nih.gov/genbank/

González G, C Comas, V Confalonieri, C A Naranjo, L Poggio (2006) Genomic affinities between maize and Zea perennis using classical and molecular cytogenetic (GISH-FISH). Chromosome Res. 14:629-635.

González G, V Confalonieri, C Comas, C A Naranjo, L Poggio (2004) GISH Genomic in situ hybridization reveals cryptic genetic differences between maize and its putative wild progenitor Zea mays subsp. parviglumis. Genome 47:947-953.

González G, Poggio L (2011) Karyotype of Zea luxurians and Z. mays subsp. mays using FISH/DAPI, and analysis of meiotic behavior of hybrids. Genome 54:26-32.
Grobman A, W Salhuana, R Sevilla (1961) Races of Maize in Perú: Their Origins, Evolution and Classification. National Academy of Sciences-National Research Council. Washington D. C., USA. $375 \mathrm{p}$.

Kato T A, C Mapes S, L M Mera O, J A Serratos H, R A Bye B (2009) Origen y Diversificación del Maíz: Una Revisión Analítica. Universidad Autónoma de México, Comisión Nacional para el Conocimiento y Uso de la Biodiversidad. México, D.F. 116 p.

Kato T A, R López (1990) Chromosome knobs of the perennial teosintes. Maydica 35:125-141.

Kato T A (1976) Cytological studies of maize (Zea mays L.) and teosinte (Zea mexicana Schrader Kuntze) in relation to their origin and evolution. Mass. Agr. Exp. St. Res. Bull. 635:1-185.

Laurie D A, M D Bennett (1985) Nuclear DNA content in the genera Zea and Sorghum: Intergeneric, interespecific and intraspecific variation. Heredity 55:307-313.

Longley A E (1938) Chromosomes of maize from North American Indians. J. Agron. Res. 56:177-195.

Matsuoka Y, Y Vigouroux, M Goodman, J Sanchez, E Buckler, J F Doebley (2002) A single domestication for maize shown by multilocus microsatellite genotyping. PNAS USA-Biol. Sci. 99:60806084.

McClintock B (1978) Significance of chromosome constitutions in tracing the origin and migration of races of maize in the Americas. Maize Breeding and Genetics. D B Walden (ed). Wiley, N.Y. pp: 59-184.

McClintock B, T A Kato, A Bluemenshein (1981) Chromosome Constitution of Races of Maize. Colegio de Postgraduados, Chapingo, México. $521 \mathrm{p}$.

Peacock W J, E S Dennis, M M Rhoades, A J Pryor (1981) Highly repeated DNA sequences limited to knob heterochromatin in maize. PNAS USA-Biol. Sci. 78:4490-4494.

Poggio L, G González, V Confalonieri, C Comas, C A Naranjo (2005) The genome organization and diversification of maize and its allied species revisited: evidences from classical and FISHGISH cytogenetic analysis. Cytogenet. Genome Res. 109:259267.

Poggio L, C A Naranjo (1990) Contenido de ADN y evolución en plantas superiores. Acad. Nac. de Cs Ex. Fís. y Nat., Buenos Aires, Monografía 5:27-37.

Poggio L, M Rosato, M Chiavarino, C A Naranjo (1998) Genome size and environmental correlations in maize. Ann. Bot. 82:115 117.

Ramírez R E, D H Timothy, E B Díaz, U J Grant (1960) Races of Maize of Bolivia. National Academy of Sciences-National Research Council. Washington D. C.159 p.

Rayburn A L, H J Price, J D Smith, J R Gold (1985) C-band heterochromatin and DNA content variation in Zea mays. Am. J. Bot. 72:1610-1617.

Roberts L M, U J Grant, E R Ramirez, W H Hathewy, D L Smith (1957) Races of Maize in Colombia. National Academy of Sciences-National Research Council Publ. 510. Bogotá. 159 p.

Rosato M, A Chiavarino, C A Naranjo, J Cámara H, L Poggio (1998) Genome size and numerical polymorphism for B- chromosome in races of maize (Zea mays ssp. mays, Poaceae). Am. J. Bot. 85:168-174.

Rozen S, H Skaletsky (2000) Primer3 on the www for general users and for biologist programmers. Methods Mol. Biol. 132:365-386. Disponible en: http://frodo.wi.mit.edu/primer3/primer3_code. html.

Tito C, L Poggio, C A Naranjo (1991) Cytogenetics studies in the genus Zea: DNA content and heterochromatin in species and hybrids. Theor. Appl. Genet. 83:58-64.

Wan Y, M Widholm (1992) Chromosome knob number of somatic cell of five inbreeds. Maize Genet. Coop. Newslett. 66:178.

Wellhausen E J, O Fuentes, A Hernández C (1957) Races of Maize in Central America. National Academy of Sciences-National Research Council. México. 138 p. 